\title{
Angular power spectrum of the FASTICACMB component from BEAST data
}

\author{
S. Donzelli*1, ${ }^{\ddagger}$ D. Maino ${ }^{1}$, M. Bersanelli ${ }^{1}$, J. Childers $^{3}$, N. Figueiredo ${ }^{4}$, P.M. Lubin ${ }^{3}$, \\ P.R. Meinhold ${ }^{3}$, I.J. O'Dwyer ${ }^{5}$, M.D. Seiffert ${ }^{6}$, T. Villela ${ }^{7}$, B.D. Wandelt ${ }^{5}$, C.A. \\ Wuensche $^{7}$ \\ ${ }^{1}$ Dipartimento di Fisica, Università di Milano, Via Celoria 16, 20133, Milano, Italy. \\ ${ }^{2}$ SISSA/ISAS, Astrophysics Sector, Via Beirut 4, 34014, Trieste, Italy. \\ ${ }^{3}$ Physics Department, University of California, Santa Barbara, CA 93106. \\ ${ }^{4}$ Universidade Federal de Itajubá, Departamento de Física e Química, Caixa Postal 50, \\ 37500-903, Itajubá, MG, Brazil. \\ ${ }^{5}$ Astronomy Department, University of Illinois at Urbana-Champaign, Urbana, IL 61801-3074. \\ ${ }^{6}$ Jet Propulsion Laboratory, Oak Grove Drive, Pasadena, CA 91109. \\ ${ }^{7}$ Instituto Nacional de Pesquisas Espaciais, Divisão de Astrofísica, Caixa Postal 515, \\ 12245-970, São José dos Campos, SP, Brazil.
}

\begin{abstract}
We present the angular power spectrum of the CMB component extracted with FASTICA from the data of the Background Emission Anisotropy Scanning Telescope (BEAST), operating in Q (38-45 GHz) and $\mathrm{Ka}(26-36 \mathrm{GHz})$ bands from the UC White Mountain Research Station. With two input channels FASTICA returns two possible independent components. We found that one of them has an unphysical spectral behaviour, due to the poor $\mathrm{S} / \mathrm{N}$ ratio, while the other is a reasonable CMB component. After a detailed calibration procedure based on Monte-Carlo (MC) simulations we extracted the angular power spectrum for the identified CMB component and found a very good agreement with the already published BEAST CMB angular power spectrum (O'Dwyer et al., 2005) computed using only the $\mathrm{Q}$ band data, and with the WMAP data.
\end{abstract}

$C M B$ and Physics of the Early Universe 20-22 April 2006

Ischia, Italy

\footnotetext{
*Speaker.

†E-mail: simona.donzelli@mi.infn.it
} 


\section{Application of FASTICA to BEAST data}

FASTICA is a blind component separation technique, that operates assuming the astrophysical signals as independent processes with non-Gaussian distribution (except at most one). We can write the data model as $\mathbf{x}(\mathbf{r})=\mathbf{A s}(\mathbf{r})+\varepsilon(\mathbf{r})$, where $\mathbf{x}$ is the vector of data observed in $N$ different frequencies, $\mathbf{s}$ is the vector of signals, $\mathbf{A}$ is the mixing matrix and $\varepsilon$ is the instrumental noise. FASTICA estimates the separation matrix $\mathbf{W}$ maximizing the non-Gaussianity of the recovered components, such that $\mathbf{y}=\mathbf{W} \mathbf{x}$ are the independent components. From $\mathbf{W}^{-1}$ we can derive the frequency scalings for each component. The FASTICA algorithm can operate in three configurations, indicated with $p, g$ and $t$, depending on the different approximation of non-Gaussianity measure chosen.

We applied FASTICA to the data of the Background Emission Anisotropy Scanning Telescope (BEAST): two annular sky maps around the $\mathrm{NCP}\left(33^{\circ}<\delta<42^{\circ}\right)$ in $\mathrm{Ka}(30 \mathrm{GHz})$ and $\mathrm{Q}(41.5 \mathrm{GHz})$ bands, with a resolution of $30^{\prime}$ and $23^{\prime}$ respectively. A study of the noise properties shows that the $\mathrm{S} / \mathrm{N}$ ratio is quiet poor $(\sim 0.11$ for the $\mathrm{Q}$ band) and the noise is not uniformly distributed on the sky [3]. Before applying FASTICA, since the approach requires maps at the same angular resolution, we have smoothed them choosing values of $30^{\prime}, 40^{\prime}$ and $60^{\prime}$. In addition we have cut the galactic plane within three different values of latitude: $|b| \leq 17.5^{\circ},|b| \leq 20^{\circ},|b| \leq 22^{\circ}$.

\section{The CMB component}

With two input maps FASTICA extracts two output maps. For all the considered cases, due to the poor $\mathrm{S} / \mathrm{N}$ ratio, one is clearly noise related while the other is an astrophysical component, but still strongly affected by noise. To verify the consistency of this second output with CMB signal we look at the frequency scaling as a first figure of merit, finding quite different values (Fig i]) from that expected for the CMB between Ka and $\mathrm{Q}$ frequencies (1.022, in antenna temperature). Nevertheless the spatial pattern resembles the Q map pattern (Fig. 1). Therefore we proceeded by verifying that reconstructed maps at different resolution have high spatial correlation one each other and with the $\mathrm{Q}$ map, characterized by a better $\mathrm{S} / \mathrm{N}$ ratio. Finally, we verified that there are not significant changes in the astrophysical component reconstruction when extending the galactic cut. This points out that the galactic contribution is not relevant. Therefore we are confident that FASTICA recovers a signal dominated by CMB anisotropies.

\section{Testing with MC simulations}

In order to test the $\mathrm{CMB}$ reconstruction quality we performed 100 Monte-Carlo simulations for each resolution in which CMB signal is simulated, observed following BEAST observing strategy and reduced as the actual data. Then we added noise realizations with the same rms per pixel and angular distribution of BEAST maps. Since after the Galactic plane cut the foreground contribution is negligible, we decided do not add any foreground templates. After applying FASTICA to the simulated maps, we considered the recovered CMB and studied the relation between the frequency scaling and correlation coefficient with the input CMB (Fig. 2). The expected frequency scaling corresponds to the higher correlation, meaning the best recovered CMB. Therefore we can select the maximum value of scaling for a "good" CMB reconstruction. Comparison with the results out 

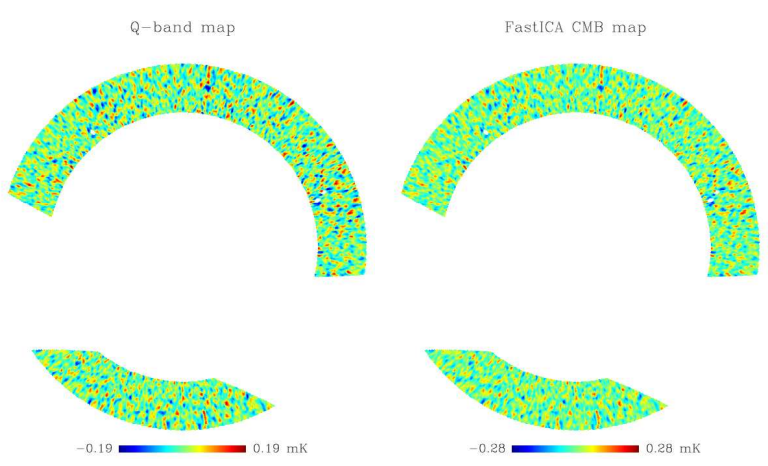

\begin{tabular}{ccccc}
\hline & cut & $p$ & $g$ & $t$ \\
$30^{\prime}$ & $|b| \leq 17.5^{\circ}$ & 0.221 & 0.129 & 2.382 \\
& $|b| \leq 20^{\circ}$ & 0.190 & 0.118 & 2.470 \\
& $|b| \leq 22^{\circ}$ & 0.177 & 0.068 & 2.480 \\
$40^{\prime}$ & $|b| \leq 17.5^{\circ}$ & 0.127 & 1.296 & 2.683 \\
& $|b| \leq 20^{\circ}$ & 1.459 & 1.116 & 3.036 \\
& $|b| \leq 22^{\circ}$ & 1.580 & 1.090 & 2.838 \\
$60^{\prime}$ & $|b| \leq 17.5^{\circ}$ & 1.049 & 1.148 & 2.917 \\
& $|b| \leq 20^{\circ}$ & 0.927 & ${ }^{*}$ & 3.141 \\
& $|b| \leq 22^{\circ}$ & 0.930 & 0.815 & 2.928 \\
\hline$*$ no convergence of FASTICA algorithm
\end{tabular}

Figure 1: left: The BEAST Q-band map and the recovered FASTICA CMB component at $40^{\prime}$; right: Frequency scalings of the FASTICA CMB component.

of BEAST data confirms that FASTICA recovers a reliable CMB component. The relation shows also the increasing reconstruction quality with angular scale, due to the increasing $\mathrm{S} / \mathrm{N}$ ratio.

\section{Normalization}

Since FASTICA does not recover a CMB signal with the correct variance, we used MC simulations to obtain a normalization procedure. In this case the scale factor is just the ratio between output and input CMB rms. For each resolution we found a clear relationship between scale factor and frequency scaling that is almost linear within certain values of scaling and normalization factor (Fig. 3). In addition CMB reconstructions with the expected frequency scaling have also the correct normalization. The scale factor for the FASTICA results out of BEAST data are derived by interpolation of this relation at the derived frequency scaling.

\section{Power spectrum}

We extracted the angular power spectrum of the FASTICA CMB component from BEAST data, choosing a $40^{\prime}$ map. We adopted a MASTER approach [2], that allows for de-biasing the power spectrum for the effects specific of the experimental observation. Since the FASTICA CMB map is a linear combination of data maps in two frequencies, Ka and Q, we took into account experimental observation effects in both the bands. Finally we normalized the power spectrum with the proper scale factor. We found (Fig. (4) a very good agreement with our results and the best-fit WMAP 1yr model and also with the spectrum estimated from the BEAST 23' Q-band map [4]. This confirms the reliability of the CMB extracted by FASTICA and validates our normalization procedure.

\section{References}

[1] S. Donzelli et al., to be published in MNRAS doi: 10.1111/j.1365-2966.2006.10320.x (2006).

[2] E. Hivon et al., Astrophys. J. , 567, 2 (2002).

[3] P.R. Meinhold et al., Astrophys. J. Supp. , 158, 101 (2005).

[4] I.J. O’Dwyer et al., Astrophys. J. Supp., 158, 93 (2005). 

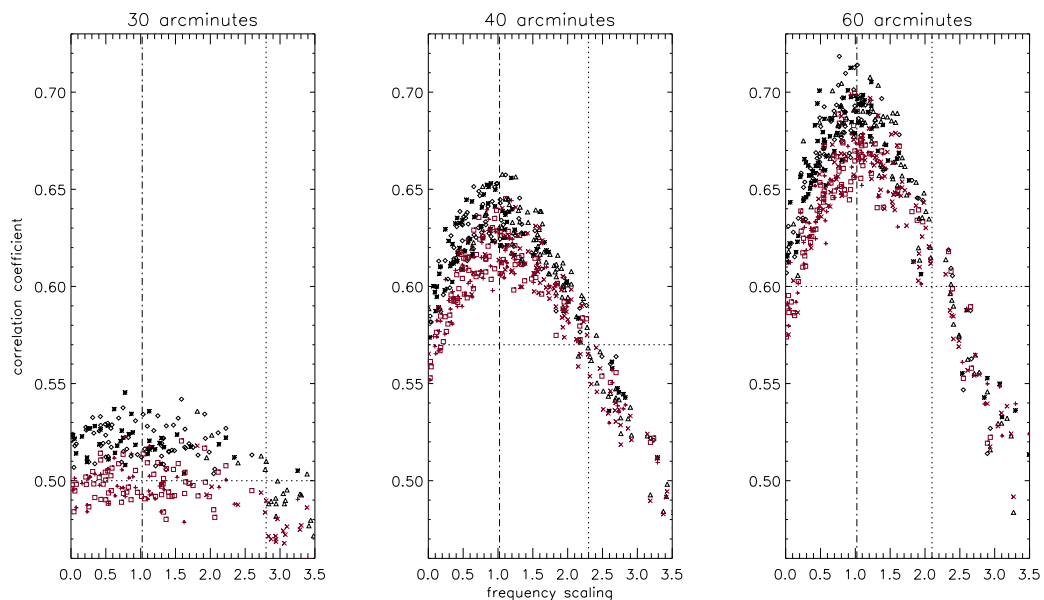

Figure 2: Correlation between ICA CMB and simulated CMB map (in red Ka, in black Q) towards recovered frequency scalings.
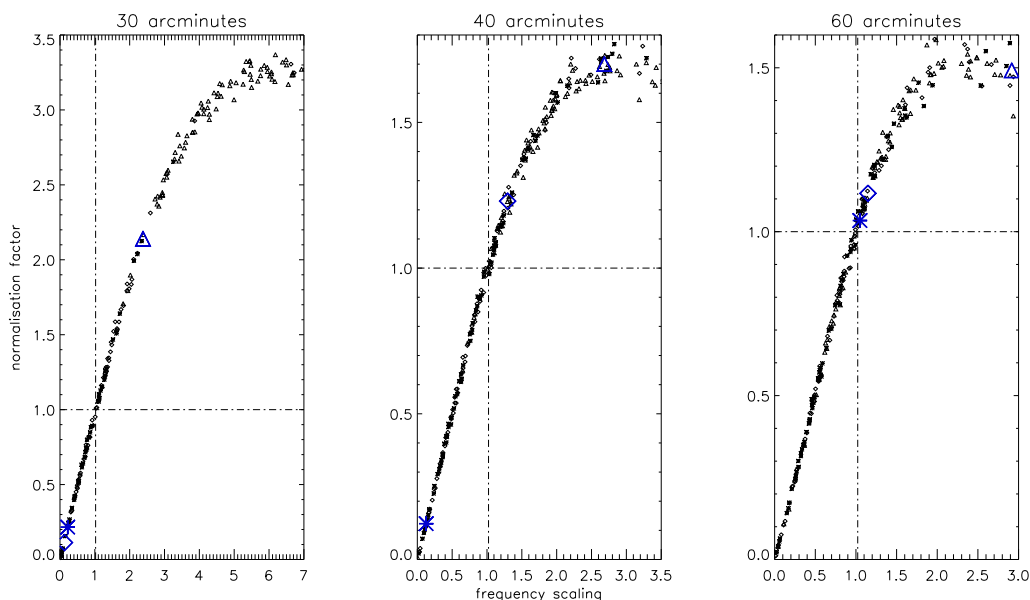

Figure 3: Normalization factor as a function of frequency scaling. The bigger symbols show the interpolation with scalings out of BEAST data (asterisk: p, diamonds: $g$, triangles: $\mathrm{t}$ ).

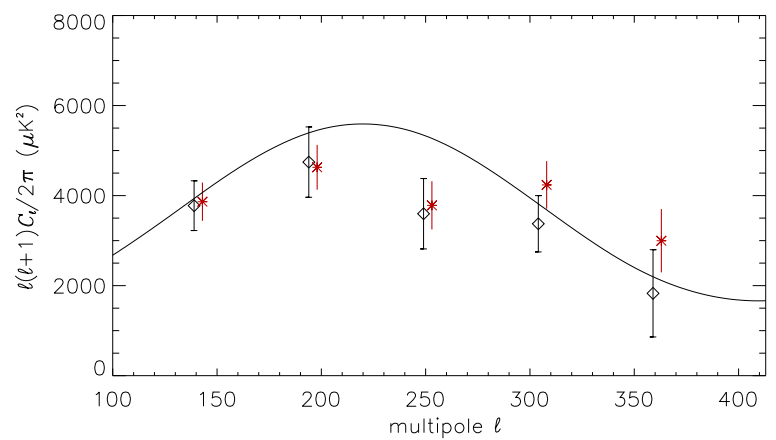

Figure 4: CMB power spectra from BEAST data: diamonds show the Q-band spectrum, stars the FASTICA CMB spectrum (shifted by $\Delta \ell=4$ for clarity). Solid line is the best-fit WMAP spectrum. 